\title{
Imperfectly grown periodic medium: absence of localized states
}

\author{
Alexander Y. Gordon
}

\begin{abstract}
We consider a discrete model of the $d$-dimensional medium with Hamiltonian $\Delta+v$; the lattice potential $v$ is constructed recursively on a nested sequence of cubes $Q_{n}$ obtained by successive inflations with integer coefficients. Initially, the potential is defined on the cube $Q_{0}$. At the $n$th step the potential, which is already constructed on the cube $Q_{n-1}$, gets extended $Q_{n-1}$-periodically to the cube $Q_{n}$; then its values at $m_{n}$ randomly chosen points of $Q_{n}$ are arbitrarily changed. This alternating process of periodic extension and introduction of impurities goes on, resulting in an (in general, unbounded) potential $v$. We show that if the size of the cube $Q_{n}$ grows fast enough with $n$ while the sequence $m_{n}$ grows not too fast, then the Schrödinger operator $\Delta+v$ almost surely does not have eigenvalues.
\end{abstract}

Mathematics Subject Classification (2010). Primary 39A70; Secondary 47H40.

Keywords. Periodic operator, random perturbation, eigenvalue, localized state.

\section{Introduction}

We consider a discrete model of the $d$-dimensional medium with Hamiltonian $h_{v}=\Delta+v$. Here $\Delta$ is the lattice Laplacian, and the lattice potential $v(\cdot)$ is the result of the following recursive process. Consider a nested sequence of cubes $Q_{n}(n=0,1,2, \ldots)$ of the form

$$
Q_{n}=\left\{x \in \mathbf{Z}^{d}:-\frac{T_{n}}{2}<x_{i} \leq \frac{T_{n}}{2}, i=1, \ldots, d\right\}
$$

whose sides $T_{n} \in \mathbf{N}$ are such that

$$
\frac{T_{n}}{T_{n-1}} \in \mathbf{N} \backslash\{1\}, \quad n=1,2,3, \ldots
$$


Initially, the potential is defined on the cube $Q_{0}$. We want to extend it $Q_{0}$-periodically to the entire lattice $\mathbf{Z}^{d}$, but we can only do that in steps: first extend it $Q_{0}$-periodically to the cube $Q_{1}$; then extend the resulting potential $Q_{1}$-periodically to the cube $Q_{2}$; etc. Assume, furthermore, that the process of periodic extension of the potential from the cube $Q_{n-1}$ to the cube $Q_{n}$ is imperfect: the values of the resulting $Q_{n-1}$-periodic potential on $Q_{n}$ are then changed arbitrarily at $m_{n}$ points chosen at random so that all such choices are equiprobable.

The potential $v$ is, therefore, "grown" by the alternating process of periodic extension and introduction of "impurities". The Borel-Cantelli lemma guarantees that $v(\cdot)$ is almost surely well-defined if

$$
\sum_{n=1}^{\infty} \frac{m_{n}}{T_{n}^{d}}<\infty
$$

In general, the potential $v$ is unbounded.

The goal of this work is to show that if the sequence $T_{n}$ grows fast enough while the sequence $m_{n}$ grows not too fast, then almost surely the operator $h_{v}$ does not have eigenvalues - in other words, there are no localized states. ${ }^{1}$ Before formulating the result precisely, we will describe our model in an equivalent but more convenient way.

We consider a Schrödinger operator in $l^{2}\left(\mathbf{Z}^{d}\right)$

$$
h_{v}=\Delta+v \text {, }
$$

where $\Delta$ is the lattice Laplacian,

$$
(\Delta y)(x)=\sum_{\|z\|_{1}=1} y(x+z), \quad x \in \mathbf{Z}^{d},
$$

and $v$ is the operator of multiplication by the function

$$
v(x)=\sum_{n=0}^{\infty} u_{n}(x) .
$$

Here $u_{n}(\cdot)$ is a $\left(T_{n}, \ldots, T_{n}\right)$-periodic function on $\mathbf{Z}^{d}$, where the numbers $T_{n} \in \mathbf{N}$ $(n=0,1,2, \ldots)$ satisfy condition (2). We assume that the function $u_{n}(\cdot)(n \geq 1)$ on its elementary cell $Q_{n}$ defined by (1) vanishes everywhere except for a subset $X_{n}$ of $Q_{n}$ which consists of $m_{n}$ points selected at random so that all such subsets of $Q_{n}$ are equiprobable. The values of $u_{n}(\cdot)$ at those points are chosen arbitrarily.

${ }^{1}$ Note that if $m_{n}=0$ for $n \geq n_{0}$, then the potential $v$ is periodic; the spectrum of $h_{v}$ in this case is well known to be not only continuous (no eigenvalues) but absolutely continuous. 
Under the condition (3), the potential $v$ is almost surely well-defined, and the operator $h_{v}$ is an (in general, unbounded) operator in $l^{2}\left(\mathbf{Z}^{d}\right)$ with the domain

$$
D\left(h_{v}\right)=\left\{y \in l^{2}\left(\mathbf{Z}^{d}\right): \sum_{x \in \mathbf{Z}^{d}}|v(x) y(x)|^{2}<\infty\right\} .
$$

In our model, the functions $u_{n}$ (and hence their sum $v$ ) are real-valued so the operator $h_{v}$ is self-adjoint. However, in what follows the functions $u_{n}$ may be complex-valued.

Let

$$
W_{n}=T_{n}^{2 d-1} \ln ^{2} T_{n}
$$

$\left(\ln ^{2} x\right.$ means $\left.(\ln x)^{2}\right)$.

\section{Theorem 1. If}

$$
\sum_{n=0}^{\infty} \frac{m_{n+1} W_{n}}{T_{n+1}}<\infty,
$$

then with probability 1 the operator (4), (5) has no eigenvalues.

Here is a more detailed formulation of Theorem 1 . By $(\Omega, \mathcal{F}, P)$ we denote the probability space of the model. Let the numbers $T_{n} \in \mathbf{N}(n \geq 0)$ satisfy the condition (2), and $X_{n}(\omega)$, for each $n \geq 1$, be a uniformly distributed random subset of cardinality $m_{n}$ of the cube (1). Under condition (6), there is a set $\Omega_{0} \in \mathcal{F}$ with $P\left(\Omega_{0}\right)=1$ and the following property: suppose $\omega \in \Omega_{0}$ and $u_{0}(\cdot), u_{1}(\cdot), u_{2}(\cdot), \ldots$ are arbitrary complex-valued functions such that each $u_{n}(\cdot)$ is $\left(T_{n}, \ldots, T_{n}\right)$-periodic and, if $n \geq 1$, vanishes on the set $Q_{n} \backslash X_{n}(\omega)$; then the operator (4), (5) does not have eigenvalues.

The proof of Theorem 1 is based on a theorem regarding solutions of periodic lattice equations. Before formulating it, we introduce some notation. For a function $y: \mathbf{Z}^{d} \rightarrow \mathbf{C}$ let

$$
\sigma(k)=\sum_{x \in \mathbf{Z}^{d}: x_{d}=k}|y(x)|^{2}, \quad k \in \mathbf{Z} \quad(0 \leq \sigma(k) \leq \infty) .
$$

Furthermore, we will represent any $T=\left\langle T_{1}, \ldots, T_{d}\right\rangle \in \mathbf{N}^{d}$ in the form

$$
T=\langle J, K\rangle,
$$

where $J=\left\langle J_{1}, \ldots, J_{d-1}\right\rangle \in \mathbf{N}^{d-1}$ and $K \in \mathbf{N}$ (so that $J_{i}=T_{i}, i=1, \ldots, d-1$, and $K=T_{d}$ ). The product $J_{1} \ldots J_{d-1}$ will be denoted by $[J]$. 
Theorem 2. For any $c>0$, there is $\gamma_{c}>0$ such that if $y: \mathbf{Z}^{d} \rightarrow \mathbf{C}$ is a solution of $(\Delta+v) y=\lambda y$, where $v(\cdot)$ is a $\left(T_{1}, \ldots, T_{d}\right)$-periodic complex-valued function on $\mathbf{Z}^{d}$, and

$$
\sigma(k)<\infty \text { for all } k \in \mathbf{Z}
$$

then

$$
\sum_{1 \leq|r| \leq \rho_{c}(J)} \sigma(r K) \geq \gamma_{c} \sigma(0),
$$

where

$$
\rho_{c}(J)=\left(c[J]^{2} \ln ^{2}[J]\right) \vee(2[J]) .
$$

Remark. Inequalities (7) hold if and only if $\sigma\left(k_{0}\right)+\sigma\left(k_{0}+1\right)<\infty$ for some $k_{0} \in \mathbf{Z}$. (This follows from inequalities (34) and (35) below.)

Corollary 1. For any $c>0$, there is $\gamma_{c}>0$ such that if $v(\cdot)$ is a $\left(T_{1}, \ldots, T_{d}\right)$-periodic function on $\mathbf{Z}^{d}$ and $y: \mathbf{Z}^{d} \rightarrow \mathbf{C}$ is a solution of $(\Delta+v) y=\lambda y$, then

$$
\sum_{K \leq|k| \leq \rho_{c}(J) K} \sigma(k) \geq \gamma_{c} \sigma(0),
$$

where $\rho_{c}(J)$ is defined by (9).

The proof of Theorem 2 is based on a lower bound for iterates of a finitedimensional linear operator.

Theorem 3. Let $A$ be an invertible linear operator in a finite-dimensional complex vector space $X$ endowed with a seminorm $\|\cdot\|$. Then for any $g>0$ and any $\xi \in X$

$$
\sum_{1 \leq|l| \leq\left(g n^{2} \ln ^{2} n\right) \vee n}\left\|A^{l} \xi\right\|^{2} \geq \mu_{g}\|\xi\|^{2},
$$

where $n=\operatorname{dim} X$ and $\mu_{g}>0$ is a constant depending only on $g$.

\section{Proofs}

2.1. Proof of Theorem 3. Our starting point is the following result that has been useful in the theory of one-dimensional Schrödinger operators. 
Lemma 1. Let $A$ be an invertible linear operator in an n-dimensional complex vector space $X,\|\cdot\|$ any seminorm on $X$, and $\xi$ any vector in $X$. Then ${ }^{2}$

$$
\max _{j= \pm 1, \ldots, \pm n}\left\|A^{j} \xi\right\| \geq \frac{1}{n}\|\xi\| .
$$

Proof (E. A. Gorin). Let $p(x)=\sum_{k=0}^{n} b_{k} x^{k}$ be the characteristic polynomial of the operator $A$, and $k_{0}\left(0 \leq k_{0} \leq n\right)$ be such that $\left|b_{k_{0}}\right|=\max _{0 \leq k \leq n}\left|b_{k}\right|$. By the Cayley-Hamilton theorem, $p(A) \xi=\sum_{k=0}^{n} b_{k} A^{k} \xi=0$, so that

$$
\xi=-b_{k_{0}}^{-1} A^{-k_{0}} \sum_{0 \leq k \leq n ; k \neq k_{0}} b_{k} A^{k} \xi=-\sum_{0 \leq k \leq n ; k \neq k_{0}}\left(b_{k} / b_{k_{0}}\right) A^{k-k_{0}} \xi,
$$

and

$$
\|\xi\| \leq \sum_{-k_{0} \leq l \leq n-k_{0} ; l \neq 0}\left\|A^{l} \xi\right\|
$$

which implies (11).

Proof of Theorem 3. In view of (12), we have

$$
\sum_{1 \leq|l| \leq n}\left\|A^{l} \xi\right\| \geq\|\xi\|
$$

Denote $\left\|A^{m} \xi\right\|$ by $a_{m}$ for all $m \in \mathbf{Z}$. We may assume that $a_{0}=1$. Pick an arbitrary integer $k \geq 1$ (its actual value will be selected later). It follows from inequality (13) applied to $A^{j}$ instead of $A$ that

$$
\sum_{l=-n}^{n} a_{l \cdot j} \geq 1, \quad j=1,2, \ldots, k
$$

(a prime following the summation symbol denotes the omission of the zero value of the summation index). For any $s \in \mathbf{Z}(s \neq 0)$, let $d_{s}$ be the number of distinct positive divisors of $s$ (including 1 and $|s|$ ). The previous inequality and the Cauchy-Schwartz inequality imply

$$
\sum_{l=-n}^{n} \frac{a_{l \cdot j}^{2}}{d_{l \cdot j}} \cdot \sum_{l=-n}^{n} d_{l \cdot j} \geq 1,
$$

${ }^{2}$ This fact was first noticed, proved and used in [2] in the case where $A$ is a unimodular operator in a two-dimensional real vector space. That was popularized by B. Simon as "Gordon's lemma". However, the proof that appeared in B. Simon's textbooks, as well as the general case of the lemma, actually belongs to E. A. Gorin (unpublished) and was communicated to B. Simon by S. Molchanov during B. Simon's visit to Moscow in 1981 (without mentioning Gorin's name). 
or

$$
\sum_{l=-n}^{n} \frac{a_{l \cdot j}^{2}}{d_{l \cdot j}} \geq\left(\sum_{l=-n}^{n} d_{l \cdot j}\right)^{-1}, \quad j=1,2, \ldots, k .
$$

Summation on $j, 1 \leq j \leq k$, gives

$$
\sum_{s=-k n}^{k n} \frac{a_{s}^{2}}{d_{s}} \cdot \Phi(s) \geq \sum_{j=1}^{k}\left(\sum_{l=-n}^{n} d_{l \cdot j}\right)^{-1},
$$

where $\Phi(s)$ is the number of representations of $s$ in the form

$$
s=l \cdot j
$$

with $-n \leq l \leq n, 1 \leq j \leq k$. Obviously, $\Phi(s) \leq d_{s}$, so that (14) implies

$$
\sum_{s=-k n}^{k n} a_{s}^{2} \geq \sum_{j=1}^{k}\left(\sum_{l=-n}^{n} d_{l \cdot j}\right)^{-1} .
$$

On the other hand, applying the inequality between the arithmetic mean and the harmonic mean

$$
\frac{1}{k} \sum_{j=1}^{k} x_{j} \geq\left(\frac{1}{k} \sum_{j=1}^{k} x_{j}^{-1}\right)^{-1} \quad\left(x_{j}>0 \text { for all } j\right),
$$

using then the obvious relation $d_{l \cdot j} \leq d_{l} \cdot d_{j}$, and putting, for any positive integer $p$,

$$
D_{p}=\sum_{s=1}^{p} d_{s},
$$

we get

$$
\begin{aligned}
\sum_{j=1}^{k}\left(\sum_{l=-n}^{n} d_{l \cdot j}\right)^{-1} & \geq k^{2}\left(\sum_{j=1}^{k} \sum_{l=-n}^{n} d_{l \cdot j}\right)^{-1} \\
& \geq k^{2}\left(\sum_{j=1}^{k} \sum_{l=-n}^{n} d_{l} \cdot d_{j}\right)^{-1} \\
& =k^{2}\left(2 D_{k} D_{n}\right)^{-1}
\end{aligned}
$$

Note that

$$
D_{p}=\sum_{i=1}^{p}\left\lfloor\frac{p}{i}\right\rfloor,
$$


where $\lfloor x\rfloor$ is the integer part of $x$. (Proof: rewrite (16) in the form

$$
D_{p}=\sum_{s=1}^{p} \sum_{i=1}^{p} y_{i s}
$$

where $y_{i s}$ equals 1 if $i$ is a divisor of $s$ and equals 0 otherwise; then reverse the order of summation. For a different proof of this simple fact, see [5, Problem VIII 79].) It follows that

$$
D_{p} \leq p \sum_{i=1}^{p} \frac{1}{i} \leq p(\ln p+1),
$$

so that (15) and (17) imply:

$$
\sum_{s=-k n}^{k n} a_{s}^{2} \geq \frac{k^{2}}{2 k n(\ln k+1)(\ln n+1)}=\frac{k}{2 n(\ln k+1)(\ln n+1)} .
$$

This is true for all integers $k \geq 1$. Now we put $k=\left\lfloor g n \ln ^{2} n \vee 1\right\rfloor$, where $g>0$. The right hand side of (18) (now depending only on $n$ ) is positive for all $n \geq 1$; as $n \rightarrow \infty$, it approaches $g / 2>0$. Therefore, it exceeds some number $\mu_{g}>0$ for all $n \geq 1$. This proves Theorem 3 .

2.2. Proof of Theorem 2. (a) Let $y(\cdot)$ be a solution of the equation

$$
(\Delta+v) y=\lambda y
$$

with a $(J, K)$-periodic $v(\cdot)$ such that $\sigma(k)<\infty$ for all $k \in \mathbf{Z}$. By including $\lambda$ in $v$ we may assume that $\lambda=0$ so that

$$
(\Delta+v) y=0 .
$$

We will use the representation of any $x \in \mathbf{Z}^{d}$ in the form $x=\langle j, k\rangle$, where $j=\left\langle j_{1}, \ldots, j_{d-1}\right\rangle \in \mathbf{Z}^{d-1}$ and $k \in \mathbf{Z}$; then (19) takes the form

$$
y(j, k+1)+y(j, k-1)+\sum_{\substack{1 \leq s \leq d-1 \\ \varepsilon= \pm 1}} y\left(j+\varepsilon e_{s}, k\right)+v(j, k) y(j, k)=0,
$$

with $j \in \mathbf{Z}^{d-1}$ and $k \in \mathbf{Z}$, and where $e_{s}=\langle 0, \ldots, 0,1,0, \ldots, 0\rangle \in \mathbf{Z}^{d-1}$ (1 is in the $s$-th place). 
(b) For $\theta=\left\langle\theta_{1}, \ldots, \theta_{d-1}\right\rangle \in \mathbf{T}^{d-1}$ let

$$
z(j, k ; \theta)=y(j, k) e^{2 \pi i(j \cdot \theta)} \quad j \in \mathbf{Z}^{d-1}, \quad k \in \mathbf{Z},
$$

where $i=\sqrt{-1}$ and

$$
j \cdot \theta=j_{1} \theta_{1}+\cdots+j_{d-1} \theta_{d-1} .
$$

Equation (20) implies that

$$
z(j, k+1)+z(j, k-1)+\sum_{\substack{1 \leq s \leq d-1 \\ \varepsilon= \pm 1}} e^{-2 \pi i \varepsilon \theta_{s}} z\left(j+\varepsilon e_{s}, k\right)+v(j, k) z(j, k)=0,
$$

with $j \in \mathbf{Z}^{d-1}$ and $k \in \mathbf{Z}$.

(c) Consider the "discrete cylinder" $C_{J}$,

$$
C_{J}=\mathbf{Z}_{J}^{d-1} \times \mathbf{Z}
$$

where

$$
\mathbf{Z}_{J}^{d-1}=\left(\mathbf{Z} / J_{1} \mathbf{Z}\right) \times \ldots \times\left(\mathbf{Z} / J_{d-1} \mathbf{Z}\right) \simeq \mathbf{Z}^{d-1} / G_{J} .
$$

Here $G_{J}$ is the following subgroup of $\mathbf{Z}^{d-1}$ :

$$
G_{J}=\left\{x=\left\langle x_{1}, \ldots, x_{d-1}\right\rangle \in \mathbf{Z}^{d-1}: x_{1} \in J_{1} \mathbf{Z}, \ldots, x_{d-1} \in J_{d-1} \mathbf{Z}\right\} .
$$

The function $v(j, k)$, being $(J, K)$-periodic, carries over in a natural manner to $C_{J}$, giving rise to a function $V(v, k)\left(v \in \mathbf{Z}_{J}^{d-1}, k \in \mathbf{Z}\right)$, which is $K$-periodic in $k$ :

$$
V(v, k+K)=V(v, k), \quad v \in \mathbf{Z}_{J}^{d-1}, k \in \mathbf{Z} .
$$

(d) For each pair $\langle v, k\rangle \in C_{J}$, define a function $Z(v, k ; \cdot) \in L^{2}\left(\mathbf{T}^{d-1}\right)$ as the sum of the series

$$
Z(v, k ; \theta)=\sum_{j \in v} z(j, k ; \theta) \equiv \sum_{j \in v} y(j, k) e^{2 \pi i(j \cdot \theta)},
$$

which converges in $L^{2}\left(\mathbf{T}^{d-1}\right)$ due to (7).

Fix $\langle v, k\rangle \in C_{J}$. Summation on $j \in v$ in (21) gives the following equation in $L^{2}\left(\mathbf{T}^{d-1}\right)$ :

$$
\begin{aligned}
& Z(v, k+1 ; \theta)+Z(v, k-1 ; \theta) \\
& \quad+\sum_{\substack{1 \leq s \leq d-1 \\
\varepsilon= \pm 1}} e^{-2 \pi i \varepsilon \theta_{s}} Z\left(v+\varepsilon e_{s}, k ; \theta\right)+V(v, k) Z(v, k ; \theta)=0 .
\end{aligned}
$$


Each $Z(v, k ; \cdot)$, as an element of $L^{2}\left(\mathbf{T}^{d-1}\right)$, is a class of equivalent square integrable Lebesgue measurable functions on $\mathbf{T}^{d-1}$; select a Borel measurable function in this class. From now on $Z(v, k ; \cdot)$ will denote that function on $\mathbf{T}^{d-1}$, and each equation (24) will be considered as a pointwise equation that holds for Lebesgue almost every $\theta \in \mathbf{T}^{d-1}$. Let $B$ be a full measure Borel subset of $\mathbf{T}^{d-1}$ such that for any $\theta \in B$ all equations (24) hold.

(e) Pick an arbitrary $\theta \in B$. For any $k \in \mathbf{Z}$, consider a vector

$$
\zeta_{k}(\theta)=\langle\mathbf{Z}(\nu, k ; \theta)\rangle_{\nu \in \mathbf{Z}_{J}^{d-1}} \in \mathcal{M},
$$

where $\mathcal{M}$ is the $[J]$-dimensional coordinate vector space over $\mathbf{C}$ with coordinates labeled by elements of $\mathbf{Z}_{J}^{d-1}$. Equation (24) can be rewritten in the form

$$
\zeta_{k+1}(\theta)=-\zeta_{k-1}(\theta)+L_{k}(\theta) \zeta_{k}(\theta)
$$

where $L_{k}(\theta)$ is the linear operator in $\mathcal{M}$ acting on vectors $\langle\zeta(v)\rangle_{\nu \in \mathbf{Z}_{J}^{d-1}} \in \mathcal{M}$ as follows:

$$
\left(L_{k}(\theta) \zeta\right)(v)=-\sum_{\substack{1 \leq s \leq d-1 \\ \varepsilon= \pm 1}} e^{-2 \pi i \varepsilon \theta_{s}} \zeta\left(v+\varepsilon e_{s}\right)-V(v, k) \zeta(v) .
$$

In view of (22), for any $k \in K$

$$
L_{k+K}(\theta)=L_{k}(\theta) .
$$

(f) Let

$$
\mathcal{L}=\mathcal{M} \oplus \mathcal{M}
$$

and

$$
\xi_{k}(\theta)=\left\langle\zeta_{k}(\theta), \zeta_{k-1}(\theta)\right\rangle \in \mathcal{L} .
$$

Equation (25) can be further transformed into

$$
\xi_{k+1}(\theta)=T_{k}(\theta) \xi_{k}(\theta),
$$

where $T_{k}(\theta)$ is the following linear operator in $\mathcal{L}$ :

$$
T_{k}(\theta)(\langle\zeta, \eta\rangle)=\left\langle-\eta+L_{k}(\theta) \zeta, \zeta\right\rangle .
$$

Again, $T_{k}(\theta)$ is $K$-periodic in $k$ :

$$
T_{k+K}(\theta)=T_{k}(\theta) .
$$


Let, furthermore,

$$
S(\theta)=T_{K-1}(\theta) T_{K-2}(\theta) \ldots T_{1}(\theta) T_{0}(\theta) .
$$

By (26), we have

$$
\xi_{r K}(\theta)=(S(\theta))^{r} \xi_{0}(\theta)
$$

for all $r \in \mathbf{Z}$.

(g) Let $\|\cdot\|$ be an arbitrary seminorm in $\mathcal{L}$. Put $g=c / 16$, where $c$ is the positive parameter in Theorem 2. By (27) and Theorem 3,

$$
\sum_{1 \leq|r| \leq \beta(J)}\left\|\xi_{r K}(\theta)\right\|^{2} \geq \mu_{g}\left\|\xi_{0}(\theta)\right\|^{2}
$$

where

$$
\beta(J)=g(2[J] \ln (2[J]))^{2} \vee(2[J]) .
$$

Note that if $[J]>1$ then $\beta(J) \leq c([J] \ln [J])^{2} \vee(2[J])=\rho_{c}(J)$, so by (28) we have

$$
\sum_{1 \leq|r| \leq \rho_{c}(J)}\left\|\xi_{r K}(\theta)\right\|^{2} \geq \mu_{g}\left\|\xi_{0}(\theta)\right\|^{2}
$$

If $[J]=1$, the inequality $\rho_{c}(J) \geq 2$ implies that

$$
\sum_{1 \leq|r| \leq \rho_{c}(J)}\left\|\xi_{r K}(\theta)\right\|^{2} \geq \sum_{1 \leq|r| \leq 2}\left\|\xi_{r K}(\theta)\right\|^{2} \geq \frac{1}{4}\left\|\xi_{0}(\theta)\right\|^{2}
$$

(the second inequality follows from Lemma 1 with $n=2$ ). Therefore, in all cases

$$
\sum_{1 \leq|r| \leq \rho_{c}(J)}\left\|\xi_{r K}(\theta)\right\|^{2} \geq \gamma_{c}\left\|\xi_{0}(\theta)\right\|^{2}
$$

where $\gamma_{c}=\left(\mu_{c / 16}\right) \wedge(1 / 4)$.

(h) Choose the following seminorm in $\mathcal{L}$ :

$$
\|\langle\zeta, \eta\rangle\|=\left(\sum_{\nu \in \mathbf{Z}_{J}^{d-1}}\left|\zeta_{\nu}\right|^{2}\right)^{1 / 2}
$$

Then (29) becomes

$$
\sum_{1 \leq|r| \leq \rho_{c}(J)}\left(\sum_{v \in \mathbf{Z}_{J}^{d-1}}|Z(v, r K ; \theta)|^{2}\right) \geq \gamma_{c} \sum_{\nu \in \mathbf{Z}_{J}^{d-1}}|Z(\nu, 0 ; \theta)|^{2},
$$


which is true for all $\theta \in B$.

Now integrate both sides of this inequality over $B$ or, equivalently, over $\mathbf{T}^{d-1}$. By Parseval's identity, (23) implies that

$$
\int_{\mathbf{T}^{d-1}}|Z(v, k, \theta)|^{2} d \theta=\sum_{j \in v}|y(j, k)|^{2},
$$

so that (31) upon integration gives

$$
\sum_{1 \leq|r| \leq \rho_{c}(J)}\left(\sum_{j \in \mathbf{Z}^{d-1}}|y(j, r K)|^{2}\right) \geq \gamma_{c} \sum_{j \in \mathbf{Z}^{d-1}}|y(j, 0)|^{2},
$$

or

$$
\sum_{1 \leq|r| \leq \rho_{c}(J)} \sigma(r K) \geq \gamma_{c} \sigma(0)
$$

This completes the proof of Theorem 2 .

Remark. The following version of Theorem 2 is true without the assumption (7).

Theorem 4. If $y: \mathbf{Z}^{d} \rightarrow \mathbf{C}$ is a solution of $(\Delta+v) y=\lambda y$, where $v(\cdot)$ is $\left(T_{1}, \ldots, T_{d}\right)$-periodic on $\mathbf{Z}^{d}$, then for any $c>0$

$$
\sum_{1 \leq|r| \leq \rho_{c}(J)}(\sigma(r K)+\sigma(r K-1)) \geq \gamma_{c}(\sigma(0)+\sigma(-1)),
$$

where $\rho_{c}(J)$ and $\gamma_{c}$ are the same as in Theorem 2.

Proof. We may assume that the left-hand side of (32) is finite. Then

$$
\sigma(K)+\sigma(K-1)<\infty .
$$

Let

$$
y_{k}=\langle y(j, k)\rangle_{j \in \mathbf{Z}^{d-1}}
$$

so that

$$
\left\|y_{k}\right\|_{2}=(\sigma(k))^{1 / 2} .
$$

In view of (20) and Minkowski's inequality,

$$
(\sigma(k+1))^{1 / 2} \leq(\sigma(k-1))^{1 / 2}+\left(2(d-1)+\|v\|_{\infty}\right)(\sigma(k))^{1 / 2}
$$

and similarly

$$
(\sigma(k-1))^{1 / 2} \leq(\sigma(k+1))^{1 / 2}+\left(2(d-1)+\|v\|_{\infty}\right)(\sigma(k))^{1 / 2},
$$


so (33) implies that $\sigma(k)<\infty$ for all $k \in \mathbf{Z}$. The remaining part of the proof of Theorem 4 is identical to the proof of Theorem 2 except for the last step when we choose, instead of (30), the standard Euclidean norm in $\mathcal{L}$,

$$
\|\langle\zeta, \eta\rangle\|=\left(\sum_{\nu \in \mathbf{Z}_{J}^{d-1}}\left|\zeta_{\nu}\right|^{2}+\sum_{\nu \in \mathbf{Z}_{J}^{d-1}}\left|\eta_{\nu}\right|^{2}\right)^{1 / 2}
$$

and, instead of (8), obtain (32).

Proof of Corollary 1. We may assume that the left-hand side of (10) is finite. Since $\rho_{c}(J) \geq 2$, we have $\sigma(K)+\sigma(K+1)<\infty$ and hence, in view of inequalities (34) and (35), $\sigma(k)<\infty$ for all $k \in \mathbf{Z}$. Now (10) follows from Theorem 2.

2.3. Proof of Theorem 1. For all $n \geq 0$ and $x \in \mathbf{Z}^{d}$, let

$$
v_{n}(x)=u_{0}(x)+\cdots+u_{n}(x) .
$$

Consider the elementary cell of $v_{n+1}(\cdot)$ :

$$
Q_{n+1}=\left\{x \in \mathbf{Z}^{d}:-\frac{T_{n+1}}{2}<x_{j} \leq \frac{T_{n+1}}{2}, j=1, \ldots, d\right\}
$$

and the set

$$
L_{n}=\left\{x \in \mathbf{Z}^{d}:-W_{n} \leq x_{d} \leq W_{n}\right\} .
$$

Let

$$
w_{n+1}=\operatorname{Pr}\left\{X_{n+1} \cap L_{n} \neq \emptyset\right\} \equiv \operatorname{Pr}\left\{X_{n+1} \cap R_{n+1} \neq \varnothing\right\},
$$

where $R_{n+1}=Q_{n+1} \cap L_{n}$. We have

$$
w_{n+1} \leq m_{n+1} \frac{2 W_{n}+1}{T_{n+1}} .
$$

Indeed, for any point $x \in Q_{n+1}$ the probability $h_{n+1}$ of the event $x \in X_{n+1}$ is $m_{n+1} / T_{n+1}^{d}$; therefore, (36) is a consequence of the following inequalities (in which $\mathbf{E}$ denotes the expectation):

$$
w_{n+1} \leq \mathbf{E}\left[\operatorname{Card}\left(X_{n+1} \cap R_{n+1}\right)\right]=\operatorname{Card}\left(R_{n+1}\right) h_{n+1} \leq\left(2 W_{n}+1\right) T_{n+1}^{d-1} h_{n+1} .
$$

By the Borel-Cantelli Lemma, (6) implies that with probability 1 there is a random number $N(\omega)$ such that $X_{n+1} \cap L_{n}=\emptyset$ for all $n \geq N(\omega)$. Consequently, for all $n \geq N(\omega)$ we have $\left(X_{n+1} \cup X_{n+2} \cup \ldots\right) \cap L_{n}=\emptyset$ so that, on the set $L_{n}$, $u_{n+1}(x)=u_{n+2}(x)=\cdots=0$ and, therefore,

$$
v(x)=v_{n}(x) \text { on } L_{n} \text { for } n \geq N(\omega) .
$$


Suppose $y(\cdot)$ is a solution of the equation $(\Delta+v) y=\lambda y$ that belongs to $l^{2}\left(\mathbf{Z}^{d}\right)$ so that

$$
\sum_{k \in \mathbf{Z}} \sigma(k)<\infty
$$

Fix temporarily some $n \geq 0$ and denote by $y_{n}(\cdot)$ the function on $\mathbf{Z}^{d}$ satisfying the equation

$$
\left(\Delta+v_{n}\right) y_{n}=\lambda y_{n}
$$

and such that $y_{n}(j, k)=y(j, k)$ for $k=-1,0$ and all $j \in \mathbf{Z}^{d-1}$. Such function $y_{n}$ exists uniquely; put

$$
\sigma_{n}(k)=\sum_{j \in \mathbf{Z}^{d-1}}\left|y_{n}(j, k)\right|^{2}, \quad k \in \mathbf{Z} .
$$

By Corollary 1 to Theorem 2 (with $J=\left\langle T_{n}, \ldots, T_{n}\right\rangle \in \mathbf{N}^{d-1}$ and $K=T_{n}$ ), for any $c>0$

$$
\sum_{T_{n} \leq|k| \leq q_{c}(n)} \sigma_{n}\left(r T_{n}\right) \geq \gamma_{c} \sigma_{n}(0),
$$

where

$$
q_{c}(n)=\left(c\left(T_{n}^{2 d-2} \ln ^{2} T_{n}^{d-1}\right) \vee\left(2 T_{n}^{d-1}\right)\right) T_{n}
$$

and $\gamma_{c}>0$. Putting $c=(d-1)^{-2}$, for large enough $n\left(n \geq N_{0}\right)$ we have $q_{c}(n)=T_{n}^{2 d-1} \ln ^{2} T_{n}=W_{n}$ so that (38) becomes

$$
\sum_{T_{n} \leq|k| \leq W_{n}} \sigma_{n}(k) \geq \gamma \sigma_{n}(0),
$$

where $\gamma=\gamma_{(d-1)^{-2}}$.

Due to (37), we have $y_{n}(x)=y(x)$ on the set $L_{n}$ if $n \geq N(\omega)$, and hence

$$
\sum_{T_{n} \leq|k| \leq W_{n}} \sigma(k) \geq \gamma \sigma(0)
$$

if $n \geq N(\omega) \vee N_{0}$. Therefore, almost surely (a.s.) ${ }^{3}$

$$
\sigma(0) \leq \gamma^{-1} \sum_{|k| \geq T_{n}} \sigma(k)
$$

for all large enough $n$; since $\sum_{k \in \mathbf{Z}} \sigma(k)<\infty$ and $T_{n} \rightarrow \infty$ as $n \rightarrow \infty$, it follows from (40) that $\sigma(0)=0$, that is, $y(j, 0)=0$ for all $j \in \mathbf{Z}^{d-1}$.

${ }^{3}$ I.e., for $P$-a.e. $\omega$ and any $v=u_{0}+u_{1}+u_{2}+\ldots$, where each $u_{n}(n \geq 1)$ vanishes on $Q_{n} \backslash X_{n}(\omega)$. 
Similarly, the function $y^{*}(j, k)=y(j, k+1)$ satisfies the equation

$$
\left(\Delta+v^{*}\right) y^{*}=\lambda y^{*},
$$

where $v^{*}(j, k)=v(j, k+1)$ for all $j, k$. The potential $v^{*}$ has the same structure as $v$, so the inclusion $y^{*} \in l^{2}\left(\mathbf{Z}^{d}\right)$ a.s. implies that $y^{*}(j, 0)=0$ for all $j$ or, equivalently, $y(j, 1)=0$ for all $j$.

Therefore, if $(\Delta+v) y=\lambda y$ and $y \in l^{2}\left(\mathbf{Z}^{d}\right)$, then a.s. $y(j, 0)=y(j, 1)=0$ for all $j \in \mathbf{Z}^{d-1}$ and hence $y \equiv 0$ on $\mathbf{Z}^{d}$. This completes the proof of Theorem 1 .

\section{Concluding Remarks}

1. The transformation of multidimensional lattice equations close to periodic ones into quasi-1D equations, in combination with lower bounds for solutions of periodic quasi-1D equations, was first used in [3], where the absence of eigenvalues for a lattice operator with a fast uniform periodic approximation was proved. This idea was then used in [1] in a different situation, where the potential is periodic in $d-1$ coordinate directions and admits a fast local periodic approximation in the remaining direction.

2. Since in the model considered above the nonzero values of $u_{n}(\cdot)$ are arbitrary, the potential $v(x)$ in Theorem 1 can grow (with respect to the distance of $x$ from the origin) arbitrarily fast on a suitable random subset of the lattice; moreover, by choosing appropriate (say, nonnegative) values of the summands $u_{n}(\cdot)$ we can make the average

$$
A(r)=\frac{\sum_{x \in B_{r}} v(x)}{\left|B_{r}\right|}
$$

of $v$ over the cube $B_{r}=\left\{x \in \mathbf{Z}^{d} \mid\|x\|_{\infty} \leq r\right\}$ grow arbitrarily fast as $r \in \mathbf{N}$ goes to $\infty$; the operator (4) will still have no eigenvalues a.s.

3. In the model above, the intersection $X_{n}(\omega) \cap Q_{n-1}$ can be nonempty, i.e., the impurities introduced during the recursive process of the "growth" of the potential can affect its portions constructed earlier. This will not occur if we modify the definition of the random set $X_{n}(\omega)$ making it a uniformly distributed subset of cardinality $m_{n}$ of the set $Q_{n} \backslash Q_{n-1}$. This change in the model does not affect the validity of Theorem 1 and requires almost no change in its proof. 
4. The model can also be modified by assuming that the function $u_{n}(\cdot)$ representing the impurities introduced at the $n$th step $(n \geq 1)$ is defined as follows: for any point $x$ of the cube $Q_{n}, u_{n}(x)$ is a random variable such that

$$
\operatorname{Pr}\left\{u_{n}(x) \neq 0\right\} \leq p_{n} .
$$

The corresponding version of Theorem 1 states: if $\sum_{n \geq 0} T_{n+1}^{d-1} W_{n} p_{n+1}<\infty$, then with probability 1 the operator (4), (5) has no eigenvalues.

5. The elementary cell of the function $u_{n}(\cdot)$ does not have to be a cube - it can be a "rectangular box"

$$
Q_{n}=\left\{x \in \mathbf{Z}^{d}:-\frac{T_{n, i}}{2}<x_{i} \leq \frac{T_{n, i}}{2}, i=1, \ldots, d\right\},
$$

where $T_{n, i} \in \mathbf{N}$ and $T_{n, i} / T_{n-1, i} \in \mathbf{N} \backslash\{1\}(i=1, \ldots, d ; n \geq 1)$. Let

$$
T_{n, \hat{i}}=T_{n, 1} \ldots T_{n, i-1} T_{n, i+1} \ldots T_{n, d}
$$

and

$$
W_{n, i}=T_{n, i}\left(T_{n, \hat{i}}\right)^{2} \ln ^{2} T_{n, \hat{i}} .
$$

If for some $i \quad(1 \leq i \leq d)$

$$
\sum_{n=0}^{\infty} \frac{m_{n+1} W_{n, i}}{T_{n+1, i}}<\infty
$$

then with probability 1 the operator (4), (5) does not have eigenvalues.

6. It may seem that the absence of localized states in the model is caused by the fast decay, as $n$ grows, of the "density" of the impurities introduced at the $n$th step. However, this is not the case. To see why, consider a deterministic version of the model, in which the $\left(T_{n}, \ldots, T_{n}\right)$-periodic function $u_{n}(\cdot)$ vanishes on the set $L_{n-1}=\left\{x \in \mathbf{Z}^{d}: \quad-W_{n-1} \leq x_{d} \leq W_{n-1}\right\}$ while its values on the set $Q_{n} \backslash L_{n-1}$ are arbitrary. The operator (4), (5) still has no eigenvalues.

7. The main results of the paper, Theorems 2 and 1, pertain to the Schrödinger operator (4), but both theorems (and their proofs) can be adapted to other finiterange lattice operators, such as $\widetilde{\Delta}+v$, where $\widetilde{\Delta}$ is the Molchanov-Vainberg Laplacian (diagonal Laplacian) [4]:

$$
(\tilde{\Delta} y)(x)=\sum_{z \in\{-1,+1\}^{d}} y(x+z), \quad x \in \mathbf{Z}^{d} .
$$


8. Although the model considered in the paper is multi-dimensional, the "imperfectly grown" potentials can also be considered in dimension one. The absence of eigenvalues in this case is guaranteed by conditions (2) and (6) with $W_{n}=2 T_{n}$, and follows from Lemma 1. Combining this with the Simon-Spencer theorem [6] (in dimension one, potentials that are unbounded on both $\mathbf{Z}_{+}$and $\mathbf{Z}_{-}$produce no absolutely continuous spectrum), we obtain yet another class of one-dimensional operators with purely singular continuous spectrum.

Acknowledgement. It is a pleasure to thank Svetlana Jitomirskaya for discussions, which helped to improve the presentation. I am also grateful to the anonymous referee for useful suggestions.

\section{References}

[1] D. Damanik, A version of Gordon's theorem for multi-dimensional Schrödinger operators Trans. Amer. Math. Soc. 356 (2004), no. 2, 495-507. MR 2022708 Zbl 1062.47038

[2] A. Y. Gordon, The point spectrum of the one-dimensional Schrödinger operator. Uspehi Mat. Nauk 31 (1976), no. 4 (190), 257-258. In Russian. MR 0458247 Zbl 0342.34012

[3] A. Y. Gordon, A sufficient condition for the continuity of the spectrum of a discrete Schrödinger operator. Funktsional. Anal. i Prilozhen. 20 (1986), no. 4, 70-71. In Russian. English transl., Funct. Anal. Appl. 20 (1986), 313-315. MR 0878048 Zbl 0623.47059

[4] S. Molchanov and B. Vainberg, Scattering on the system of the sparse bumps: multidimensional case. Appl. Anal. 71 (1999), no. 1-4, 167-185. MR 1690097 Zbl 1022.47510

[5] G. Polya and G. Szegö, Problems and theorems in analysis. Vol. II. Theory of functions, zeros, polynomials, determinants, number theory, geometry. Revised and enlarged translation by C. E. Billigheimer of the $4^{\text {th }}$ German edition.Die Grundlehren der Mathematischen Wissenschaften, 216. MR 0396134 Zbl 0311.00002

[6] B. Simon and T. Spencer, Trace class perturbations and the absence of absolutely continuous spectra. Comm. Math. Phys. 125 (1989), no. 1, 113-125. MR 1017742

Zbl 0684.47010

Received September 3, 2013; revised November 30, 2013

Alexander Y. Gordon, Department of Mathematics and Statistics, University of North Carolina at Charlotte, 9201 University City Blvd, Charlotte, NC 28223, U.S.A.

e-mail: aygordon@uncc.edu 